\title{
Le dialogue microbiote-cellules souches
}

$>$ Le microbiote intestinal est un des plus denses et complexes du corps humain. Son impact sur la physiologie de l'hôte s'exerce à la fois localement, où il joue d'importantes fonctions métaboliques et de consolidation de la barrière intestinale, et à l'échelle systémique de l'organisme, puisqu'il influence des processus aussi fondamentaux que la croissance ou la maturation du système immunitaire. Une caractéristique de l'épithélium intestinal est son extraordinaire capacité de régénération, qui repose sur la présence de cellules souches résidant dans le fond des cryptes de Lieberkühn. Plusieurs études ont montré que le microbiote intestinal participait à ce processus de régénération, suggérant qu'il peut influencer la capacité proliférative des cellules souches intestinales (CSI). Les cellules immunitaires résidant dans l'intestin sont des médiateurs importants du dialogue microbiote-épithélium. Le microbiote peut également exercer des fonctions régulatrices sur les CSI au moyen de modes de communication plus directs, passant par exemple par la liaison de ligands bactériens aux récepteurs de l’immunité innée exprimés par les CSI. Dans cette revue, nous explorons les différents modes de communication qui peuvent s'établir entre les cellules souches et le microbiote, et leur impact sur l'homéostasie intestinale. <

\section{Le microbiote intestinal}

Le microbiote est un écosystème complexe formé par l'ensemble des microorganismes (bactéries, champignons, virus) qui résident dans un site corporel donné, comme la peau ou la cavité orale, avec lesquels il entretient une relation de symbiose mutuelle. Le plus étudié, et de loin le plus abondant et divers en microorganismes, est le microbiote intestinal,

\section{Un élément clé pour la régénération intestinale}

Aline Stedman ${ }^{1}$, Giulia Nigro, Philippe J. Sansonetti ${ }^{1,2}$

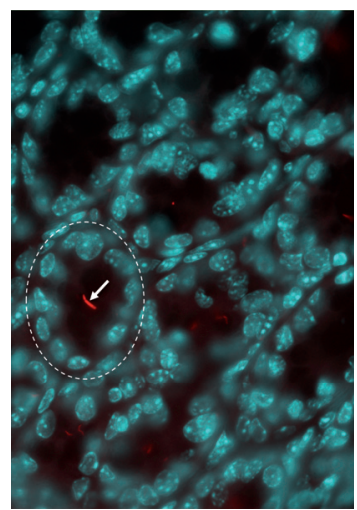

${ }^{1}$ Institut Pasteur, Unité de Pathogénie Microbienne Moléculaire, Inserm U1202, 28 , rue du Docteur Roux, 75015 Paris, France;

${ }^{2}$ Collège de France, Chaire de Microbiologie et Maladies Infectieuses, 11, place Marcelin Berthelot, 75005 Paris, France. philippe.sansonetti@pasteur.fr

surtout colique. Sa composante bactérienne est dominante et se compose principalement de bactéries appartenant aux phylums Bacteroidetes et Firmicutes. Se situant à l'interface entre aliments et épithélium, le microbiote intestinal joue un rôle primordial pour le métabolisme de l'hôte. Les bactéries du microbiote intestinal facilitent la digestion d'aliments non assimilables pour les cellules épithéliales telles que les fibres alimentaires, synthétisent de nombreux métabolites comme des vitamines (vitamines B et $K$ ) ou dégradent des agents carcinogènes comme les nitrosamines. En occupant la lumière intestinale, les bactéries commensales jouent également un rôle protecteur vis-à-vis de l'invasion par des pathogènes [1]. Enfin, le microbiote structure la barrière intestinale en régulant ses propriétés physiques, telles que la composition et l'épaisseur des couches de mucus qui tapissent l'épithélium [2], ou les jonctions serrées des cellules épithéliales [3]. II régule également le développement du système immunitaire muqueux $[4,47](\rightarrow)$.

$(\rightarrow)$ Voir la Synthèse de V. Gaboriau-Routhiau et N. Cerf-Bensussan, page 961 de ce numéro L'impact du microbiote intestinal n'est pas limité à l'intestin, il s'exerce également à l'échelle systémique des organismes hôtes. II régule en effet la maturation du système immunitaire $[5,6]$, la croissance post-natale $[7,8]$, et peut même influencer l'activité cérébrale $[9,10,48](\rightarrow)$. Chez l'homme, des perturbations du micro-

$(\rightarrow)$ Voir la Nouvelle de A.L. Barbotin et al., page 930 de ce numéro biote intestinal sont associées à une grande variété de pathologies d'ordre métabolique, immunitaire ou même cognitif $[11,12]$. 


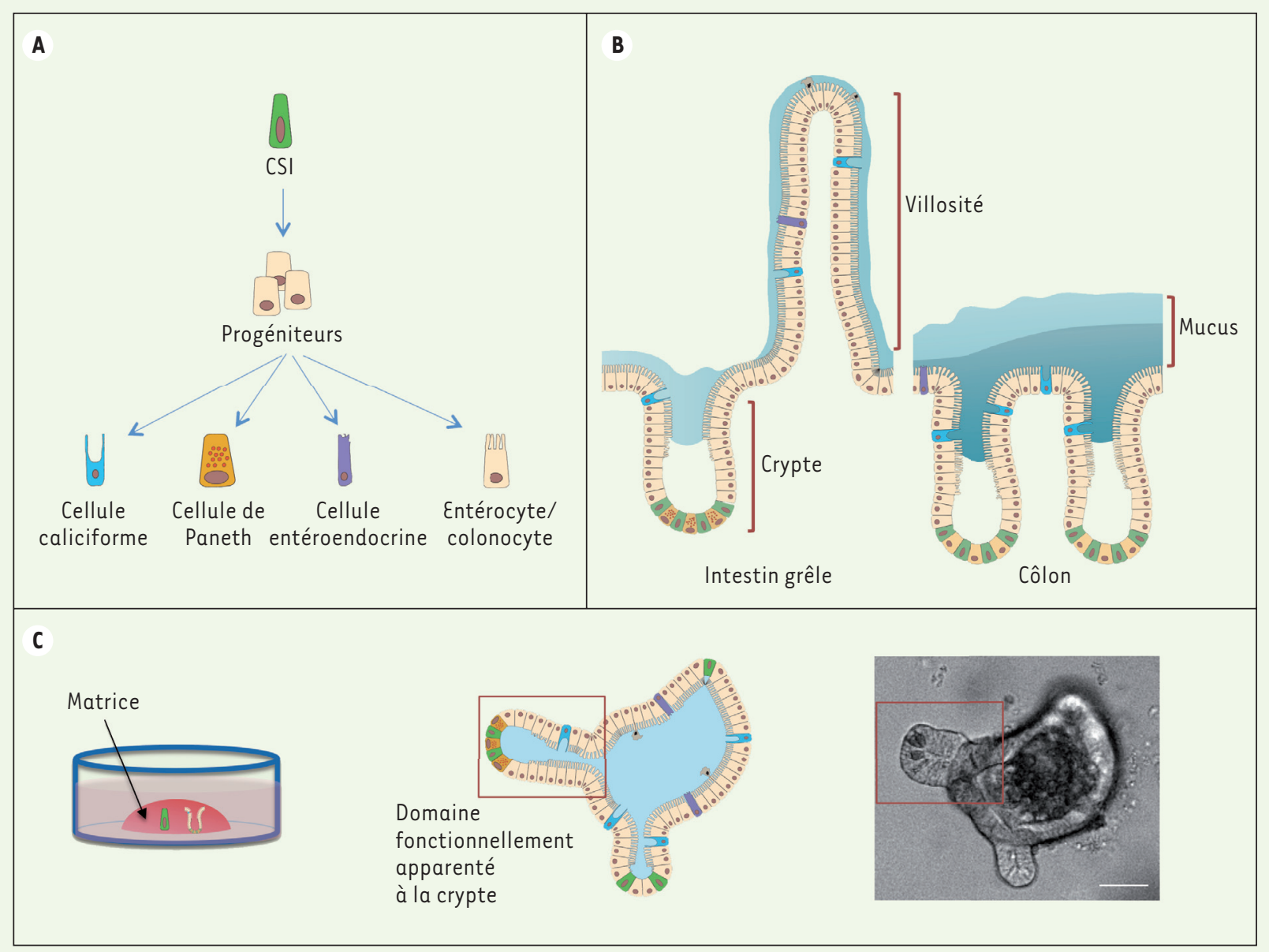

Figure 1. Les cellules souches intestinales. A. Schéma simplifié du lignage intestinal. Les cellules souches intestinales (CSI) donnent naissance à des progéniteurs qui, après plusieurs divisions, se différencient pour générer les différents types cellulaires de l'intestin. $B$. Schéma représentant l'organisation fonctionnelle de l'épithélium intestinal. Le code couleur est identique à celui du panneau A. Les cellules souches et progéniteurs intestinaux sont localisés au fond des cryptes. Au cours de leur différenciation, les cellules du lignage intestinal migrent en haut des cryptes. Dans l'intestin grêle, les cellules différenciées rejoignent les villosités. Seules les cellules de Paneth et leur équivalent colique persistent au fond de la crypte où elles restent étroitement associées aux CSI. C. Illustration de la technique de culture des organoïdes intestinaux. À gauche : les cellules souches intestinales ou les cryptes entières peuvent être isolées et mises en culture dans une matrice pour former des organoïdes intestinaux. À droite : Schéma et photographie d'un organoïde d'intestin grêle murin. Ces organoïdes sont des structures tri-dimensionnelles comprenant des régions fonctionnellement identiques aux cryptes, où résident les cellules souches et progéniteurs, et des régions intermédiaires essentiellement composées de cellules différenciées. La barre d'échelle représente $20 \mu \mathrm{m}$.

\section{Microbiote et régénération intestinale}

Une propriété de l'épithélium intestinal est son extraordinaire capacité de régénération, puisque ce tissu est entièrement renouvelé tous les 4 à 5 jours chez les mammifères, et est capable de se régénérer rapidement suite à des altérations provoquées par différents agents chimiques ou pathogènes. Cette capacité de régénération repose en grande partie sur la présence de cellules souches intestinales (CSI) à fort potentiel prolifératif. Chez les mammifères, ces cellules résident dans le fond des cryptes de Lieberkühn, qui sont des invaginations de l'épithélium, et sont caractérisées, entre autres, par l'expression du gène Lgr5 (leucine-rich repeat containing $G$ protein-coupled receptor 5 ) identifié par l'équipe de Hans Clevers [13]. Les CSI prolifèrent activement et donnent naissance à des cellules progénitrices qui, après plusieurs cycles de divisions cellulaires, se différencient pour donner l'ensemble des cellules appartenant aux quatre lignages principaux de l'intestin: les entérocytes/colonocytes, les cellules entéroendocrines, les cellules sécrétrices de mucus et les cellules de Paneth [49] $(\rightarrow)$. $(\rightarrow)$ Voir la Nouvelle de B. Romagnolo, $\mathrm{m} / \mathrm{s}$ $n^{\circ} 12$, décembre 2012, page 1058 
La différenciation des cellules est couplée à leur migration hors des cryptes, de sorte que ces cryptes forment l'unité de prolifération de l'intestin. La balance entre autorenouvellement et différenciation des CSI est finement régulée pour maintenir l'intégrité de l'épithélium et fait intervenir différents signaux émanant de leur environnement. Ainsi, dans l'intestin grêle, les cellules de Paneth sont des composants essentiels de la niche des CSI. Elles sont physiquement entremêlées aux CSI dans le fond des cryptes. Elles expriment des ligands des voies Wnt et Notch nécessaires au maintien dans l'état souche des CSI [14]. La complexité de la crypte intestinale peut être récapitulée ex vivo, grâce à un système de culture des CSI en organoïdes $^{1}$ mis au point par le laboratoire de Hans Clevers [15] (Figure 1).

Plusieurs études ont démontré que le stimulus microbien joue un rôle essentiel dans la régénération intestinale. Le dialogue entre le microbiote et la muqueuse intestinale repose en grande partie sur les récepteurs de l'immunité innée, ou pattern recognition receptors (PRR), exprimés par les cellules épithéliales et les cellules immunitaires au niveau de l'intestin [16]. Les PRR reconnaissent sélectivement des composants bactériens conservés et partagés par différents groupes de micro-organismes. Différentes familles de PRR ont été identifiées, dont les TLR (toll-like receptors) ou les NLR (NOD[nucleotide oligomerization domain]-like receptors) qui sont soit transmembranaires, soit cytosoliques. La liaison des PRR à leur ligand microbien entraîne une cascade de signalisation intracellulaire aboutissant généralement à une réponse de type inflammatoire via l'activation du facteur de transcription NFKB (nuclear factorkappa $B$ ) responsable de l'expression des cytokines pro-inflammatoires telles que I'IL(interleukine)- $1 \beta$, I'IL-6, ou I'IFN $\gamma$ (interféron $\gamma$ ) (Figure 2). L'utilisation d'animaux déficients pour ces récepteurs dans des modèles de lésions intestinales a permis de mettre en évidence le rôle essentiel du dialogue entre le microbiote et la muqueuse intestinale dans sa régénération. Ainsi, l'étude de RakoffNahoum et al. a montré que la signalisation des TLR était nécessaire à la réparation intestinale après induction de lésions par un traitement chimique au sulfate de dextran (DSS) ${ }^{2}$ [17]. En greffant la moelle osseuse de souris déficientes pour la signalisation TLR dans des souris contrôles, traitées a posteriori avec du DSS, l'équipe de Thaddeus Stappenbeck a montré que les macrophages activés localisés à la base des cryptes, participaient au mécanisme de réparation [18]. Plus récemment, les travaux menés par notre groupe ainsi que ceux réalisés par Zanello et ses collaborateurs, ont montré que le récepteur intracellulaire Nod2, de la famille des NLR, est impliqué dans la réparation de l'épithélium intestinal murin après traitement par la doxorubicine, ou après induction d'une inflammation aiguë. Là aussi, les composantes immunitaires et épithéliales de la signalisation Nod2 participent au processus de réparation [19,20]. Enfin, dans un modèle in vitro de cicatrisation cellulaire, la stimulation

\footnotetext{
${ }^{1}$ Ensemble tridimensionnel de cellules cultivées in vitro présentant les fonctions de l'organe qu'elles composent.

${ }^{2}$ Un modèle d'induction d'inflammation induite chimiquement.
}

des cellules épithéliales par la bactérie commensale Lactobacillus rhamnosus accélère la cicatrisation via l'activation de FAK (focal adhesion kinase), une kinase essentielle à la prolifération et à la migration cellulaire [21].

Le microbiote joue donc un rôle dans différents aspects du processus de restitution intestinale comme la prolifération ou la migration cellulaire. Cette fonction semble impliquer le dialogue du microbiote intestinal avec le système immunitaire et avec l'épithélium luimême.

Le microbiote intestinal est également impliqué dans le renouvellement épithélial à l'homéostasie. En effet, un raccourcissement du cycle cellulaire, ainsi qu'une diminution de la prolifération cellulaire, ont été observés chez différents animaux axéniques, des animaux stériles dépourvus de tout micro-organisme [22-24]. Compte tenu du rôle central que jouent les CSI dans la régénération intestinale, ces études indiquent que leur activité semble être modulée par le microbiote. Par quel mécanisme le dialogue entre CSI et microbiote est-il établi, et quelles sont les conséquences de ce dialogue sur la biologie des CSI?

\section{Microbiote et CSI : une communication relayée par l'environnement des CSI ?}

La drosophile constitue une version simplifiée du système intestin-microbiote et a ainsi permis d'apporter des réponses à ces questions $[25,50](\rightarrow)$.

Le microbiote intestinal de Droso-

$\rightarrow$ Voir la Nouvelle de G. Storelli et $F$. Leulier, page 925 de ce numéro phila melanogaster ne comprend en effet qu'une dizaine d'espèces bactériennes [26]. Son épithélium intestinal est formé de 2 lignages principaux : les entérocytes et les cellules entéroendocrines. II ne comporte pas de cryptes. Les cellules souches multipotentes, à l'origine de ces 2 lignages, sont donc dispersées à travers l'épithélium [27]. Plusieurs études réalisées chez la drosophile ont montré que le microbiote intestinal jouait un rôle important dans la régulation de la prolifération des CSI [25]. Cette régulation semble en grande partie relayée par les entérocytes qui, en réponse au stimulus bactérien, activent la prolifération des CSI via la signalisation JAK/ STAT (Janus kinases/signal transducers and activators of transcription) $[28,29]$, ou la signalisation Redox (oxydo-réduction), à la suite de l'activation de NOXI (NADPH oxydase 1), un complexe enzymatique membranaire exprimé par les cellules épithéliales et responsable de la libération de ROS (reactive oxygen species) [30]. Le pic oxydatif résultant de l'activation de NOXl a également été impliqué dans la cytoprotection des CSI. Le 


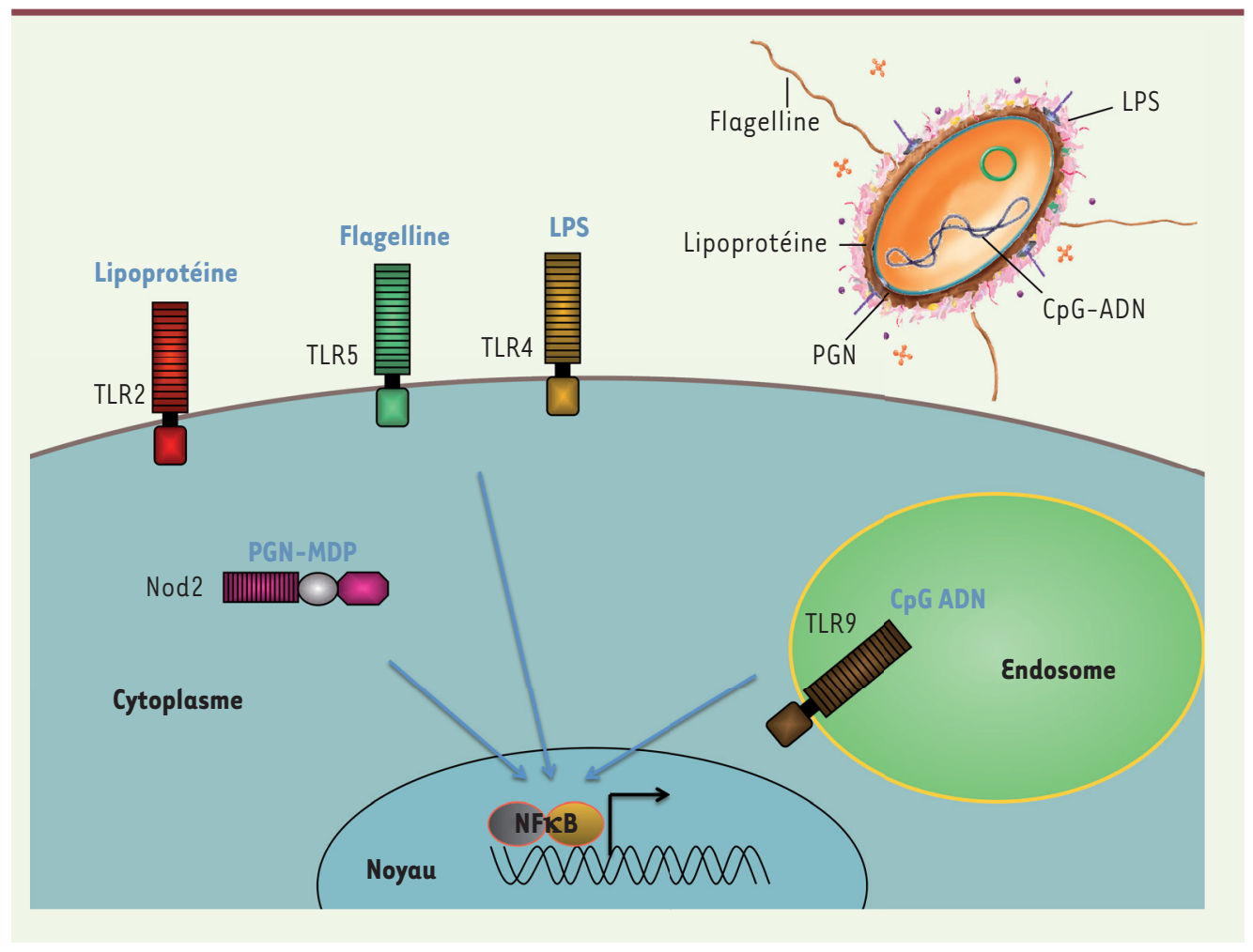

Figure 2. Les récepteurs de l'immunité innée permettent d'établir un dialogue entre le microbiote et les cellules épithéliales. Les récepteurs de l'immunité innée (PRR) reconnaissent sélectivement des composants microbiens conservés et partagés par différents groupes de micro-organismes. La liaison des PRR à leur ligand entraîne une cascade de signalisation intracellulaire pouvant aboutir à l'activation du facteur de transcription $N F \kappa B$. Les PRR sont exprimés à la fois par les cellules immunitaires et épithéliales. Sur ce schéma, seuls sont présentés quelquesuns des récepteurs reconnaissant des ligands bacté-

riens, et exprimés par les CSI (cellules souches intestinales). L'activation de la voie canonique impliquant NFKB n'a pas été démontrée dans ces cellules. TLR : toll-like receptor; LPS : lipopolysaccaride; PGN : petidoglycane ; MDP : muramyl-dipeptide ; CpG : CG dinucleotide.

microbiote pourrait donc jouer un rôle dans la protection des CSI contre les effets délétères de stress oxydatifs abiotiques ${ }^{3}[31]$. Cette fonction de cytoprotection semble conservée chez la souris [19, 31]. De même, le rôle du microbiote sur la signalisation JAK/STAT dans les CSI est conservé chez la souris et semble jouer un rôle essentiel dans la régénération épithéliale, en activant leur prolifération. Une étude récente montre en effet qu'en réponse au stimulus microbien, les lymphocytes innés de l'intestin sécrètent de l'IL-22 qui induit l'activation de STAT3 au sein des CSI. L'addition de cette cytokine à des cultures d'organoïdes murins, de même que le traitement de souris avec la cytokine, suffisent à promouvoir la prolifération des CSI [32].

\section{Une communication directe entre les CSI et le microbiote est- elle possible?}

La vision qui a prévalu pendant longtemps était que le microbiote restait séquestré au sein de la lumière intestinale, séparé de l'épithélium par le mucus. La cellule souche qui, chez les mammifères, est confinée au fond des cryptes, semblait également maintenue à distance du microbiote. On sait maintenant que des composants du microbiote peuvent établir un dialogue étroit avec la crypte intestinale, notamment dans certains cas pathologiques. Ainsi, un transfert du microbiote de la lumière intestinale

${ }^{3}$ Impropre à la vie. vers l'épithélium, a par exemple été rapporté dans des cas de maladies inflammatoires chroniques de l'intestin (MICI) $[33,34,51,52](\rightarrow)$ ou à la suite d'un régime pauvre en $(\rightarrow)$ Voir la Nouvelle de B. Lamas et al., et la Synthèse de 0 . Rahmouni et al., pages 933 et 968 de ce numéro tel rapprochement dans des cas d'altérations de la barrière intestinale, comme au cours d'un cancer colorectal, ce qui pourrait alors entraîner l'induction de signaux nécessaires à sa réparation [17].

Chez la souris, notre équipe a identifié des bactéries résidant, à l'homéostasie, dans les cryptes du cæcum et du côlon proximal [36]. Cette flore spécifique des cryptes est composée en majorité de bactéries aérobies strictes non fermentatives, appartenant en particulier au genre Acinetobacter. Des bactéries présentant des propriétés biologiques similaires sont également présentes dans la flore intestinale restreinte de la drosophile, suggérant la sélection par co-évolution d'espèces bactériennes qui résident au contact de la muqueuse intestinale lui conférant un avantage sélectif en favorisant, par exemple, la régénération épithéliale nécessaire à son homéostasie. L'ensemble de ces données indique qu'un dialogue privilégié entre la cellule souche 

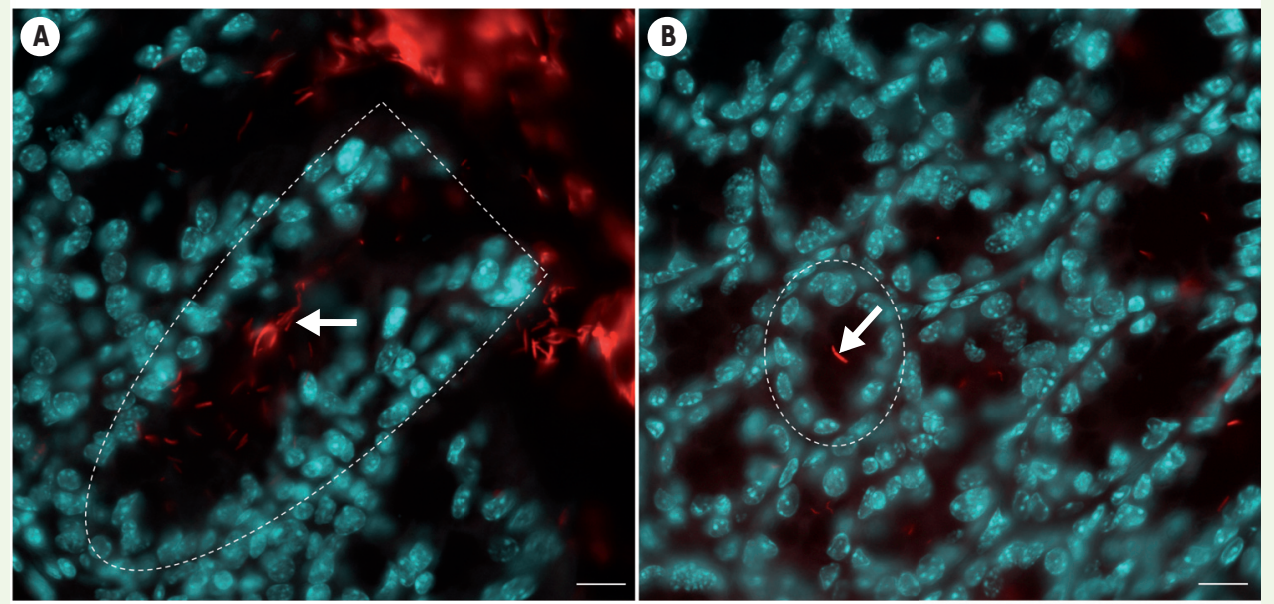

Figure 3. Une flore bactérienne spécifique des cryptes coliques. Photographies de sections longitudinales (A) ou transversales (B) de cryptes coliques murines. Les bactéries intestinales sont détectées par hybridation in situ fluorescente, en utilisant une sonde spécifique de I'ARN ribosomique bactérien 16S (marquage rouge). Les noyaux des cellules épithéliales sont marqués au DAPI (bleu). Les flèches blanches indiquent des bactéries localisées à l'intérieur des cryptes, délimitées par des traits pointillés blancs. La barre d'échelle représente $10 \mu \mathrm{m}$.

type résulte d'un effet cytoprotecteur du MDP bactérien qui est exercé directement sur les CSI. De plus, le gavage de souris par du MDP leur confère une protection contre le stress oxydatif induit par la doxorubicine, et cet effet est aboli chez les souris Nod2KO n'exprimant pas Nod2. À l'homéostasie, les cryptes de ces souris sont indifférenciables de celles des souris témoins, ce qui suggère que l'effet protecteur du microbiote ne se manifeste qu'en cas de rupture de cette homéostasie [19].

et le microbiote intestinal est possible. Par quels moyens de communication s'établit ce dialogue, et quelles en sont les conséquences sur les propriétés des CSI ? Le modèle des organoïdes intestinaux a beaucoup bénéficié aux recherches dans ce domaine, en partie parce qu'il permet de tester l'effet de différents ligands microbiens sur la crypte intestinale en s'affranchissant des effets ayant pour origine la réponse immunitaire.

\section{Une communication via les récepteurs de l'immunité innée}

Chez la souris, les CSI expriment constitutivement certains récepteurs de type PRR, dont le TLR4. La stimulation du récepteur TLR4 par son ligand bactérien, le lipopolysaccharide (LPS), soit ex vivo par stimulation d'organoïdes intestinaux, soit in vivo en injectant le LPS aux souris, induit une mort cellulaire par apoptose dans les cryptes intestinales, qui est associée à une diminution de la prolifération cellulaire, dépendant de la protéine PUMA (p53 upregulated modulator of apoptosis) [37]. Tirant parti d'un modèle de souris leur permettant de supprimer le gène TLR4 de manière mosaïque ${ }^{4}$ dans l'épithélium, les auteurs de cette étude ont pu déterminer que seules les cryptes exprimant TLR4, et non les cryptes mutantes pour ce récepteur, présentaient ce phénotype suite à la stimulation par le LPS, excluant qu'un processus de type inflammatoire puisse en être l'origine. Nous avons montré que le récepteur Nod2, qui reconnaît le muramyl-dipeptide (MDP), un fragment du peptidoglycane composant la paroi bactérienne, était exprimé plus fortement dans la CSI. La stimulation par le MDP de cryptes intestinales murines avant leur mise en culture, entraîne une forte hausse du nombre d'organoïdes formés. Nous avons montré que ce phéno-

${ }^{4} \mathrm{Ne}$ touchant pas toutes les cellules.

D'autres modes de communication entre la cellule souche intestinale et les bactéries restent à identifier. Une des caractéristiques de la flore intestinale est son importante activité métabolique. On peut donc supposer que le résultat de cette activité peut avoir un impact direct ou indirect sur les cellules souches.

\section{Les métabolites bactériens : d'importants médiateurs du dialogue microbiote-cellule souche}

L'activité métabolique de la flore intestinale est à l'origine de la libération, dans la lumière intestinale, d'une grande quantité de métabolites capables, pour certains, de rejoindre la circulation sanguine et ainsi d'interférer avec la physiologie de I'hôte $[38,53](\rightarrow)$. Une famille de récepteurs couplés à la protéine G (GPR, aussi appelés sen-

$\rightarrow$ Voir la Synthèse de R. Burcelin et al. page 952 de ce numéro seurs métaboliques), qui reconnaissent certains de ces métabolites, a été identifiée [39] ; les métabolites bactériens pourraient donc jouer un rôle dans le dialogue entre CSI et microbiote intestinal. Dans le but d'identifier des métabolites potentiellement impliqués dans le contrôle de la prolifération des CSI, Kaiko et ses collaborateurs ont exposé des cultures d'organoïdes de côlon, exprimant une luciférase sous le contrôle d'un régulateur du cycle cellulaire, à une banque de métabolites bactériens. Ils ont pu ainsi mettre en évidence l'effet anti-prolifératif du butyrate sur les CSI de côlon [40]. Le butyrate fait partie des acides gras à chaînes 


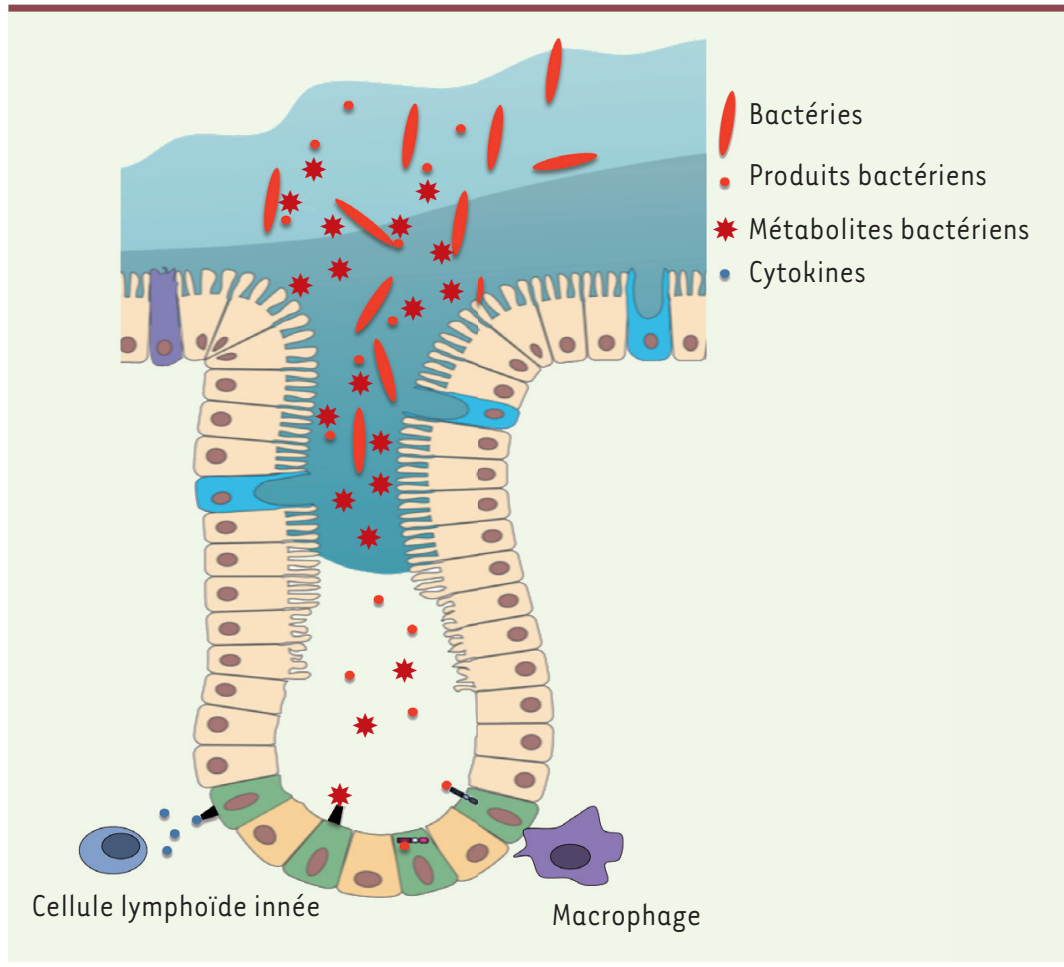

Figure 4. Le dialogue microbiote-CSI. Schéma récapitulant les interactions possibles entre le microbiote et les CSI (cellules souches intestinales). Les CSI expriment des récepteurs de l'immunité innée activés par différents ligands bactériens ainsi que des récepteurs pour certains métabolites bactériens. Les cellules immunitaires de la lamina propria, comme les cellules lymphoïdes innées et les macrophages représentés sur ce schéma, permettent également de relayer le stimulus microbien via la sécrétion de cytokines. Le code couleur des cellules est identique à celui de la Figure 1.

biote. Les marques épigénétiques incluent des modifications des histones, comme leur acétylation généralement associée à un état transcriptionnellement actif des gènes, ou la méthylation de l'ADN plutôt associée à une répression de l'expression génique. L'activité d'enzymes impliquées dans ces deux types de modifications peut être modulée par le microbiote. En effet, la diminution de la

courtes (AGCC) produits par les bactéries anaérobies coliques, lors de la fermentation des carbohydrates et des fibres alimentaires qui ne sont pas métabolisées par l'hôte. II avait déjà été impliqué dans la maturation du système immunitaire et dans la protection vis-à-vis du cancer colorectal [39]. La diminution du butyrate in vivo n'ayant pas d'effet sur la prolifération des CSI, I'hypothèse que leur localisation au sein des cryptes intestinales prévenait leur exposition à ce métabolite a été émise. Pour tester cette hypothèse, du butyrate a été administré à des poissons zèbres, dont la muqueuse intestinale est dépourvue de cryptes, mais également à des souris traitées au DSS qui, induisant une rupture de la barrière intestinale, rend les cryptes plus exposées au contenu de la lumière. Dans les deux cas, une baisse de la prolifération épithéliale a été observée. De plus, les colonocytes métabolisent activement le butyrate, ce qui fait chuter sa concentration dans la crypte, expliquant, en partie, la raison pour laquelle, à l'homéostasie, les cellules souches n'y sont pas sensibles.

Il est possible d'imaginer que d'autres métabolites bactériens puissent influencer l'autorenouvellement des CSI. Notre équipe a par exemple montré récemment que le lactate et l'acétate qui sont produits par l'activité fermentaire de Lactobacillus casei et Bifidobacterium breve, des symbiontes intestinaux, régulaient négativement le cycle cellulaire de cellules épithéliales en culture [41].

\section{Du microbiote au noyau de la cellule souche intestinale}

Le microbiote peut donc influencer le destin des CSI. Chez la drosophile comme chez la souris, le transcriptome des cellules épithéliales est fortement modulé par les stimulus microbiens [42, 43]. L'activité de certains modificateurs épigénétiques est également altérée par le micro- prolifération et la réduction du nombre de cellules de Paneth, observées chez les souris mutantes pour le gène codant l'histone déacétylase HDAC3, sont restaurées lorsque ces souris sont élevées en condition axénique [44]. De même, l'effet anti-prolifératif du butyrate décrit par Kaiko et al., reposerait sur l'inhibition de l'activité HDAC dans les CSI [40]. Chez la souris, la maturation intestinale au cours de la période postnatale est corrélée à une augmentation de la méthylation de I'ADN, en particulier grâce à l'activité de l'ADN méthylase Dnmtl (DNA [cytosine-5]-methyltransferase 1). Chez les souris axéniques, l'établissement de ce patron de méthylation est fortement perturbé, ce qui pourrait expliquer, en partie, les défauts de maturation des cryptes intestinales décrits chez ces animaux [45]. Le dialogue hôte-microbiote pourrait donc avoir un impact sur l'ontogénèse de la CSI et la maturation intestinale. Cet impact pourrait être hérité par l'ensemble du lignage intestinal et perdurer au sein de la cellule souche au cours de ses divisions. II reste maintenant à disséquer l'identité et la fonction des gènes influencés par ces régulations, et à déterminer quels composants du microbiote intestinal sont requis plus particulièrement pour l'établissement de ces marques épigénétiques.

\section{Conclusion}

L'ensemble des résultats décrits dans cette revue, en particulier les travaux récents menés chez la souris, 
mettent en évidence que le microbiote peut directement influencer la survie ou la prolifération des CSI. Différents types de stimulus, comme des composants de la paroi bactérienne ou des métabolites bactériens, ont été impliqués dans cette régulation. Les effets induits par cette stimulation peuvent varier en fonction du contexte. En effet, la signalisation par le TLR4 promeut l'autorenouvellement des CSI en cas de réparation intestinale. Mais elle induit une diminution de leur prolifération à I'homéostasie. De même, différentes réponses cellulaires sont activées selon le type de stimulus bactérien: alors que le peptidoglycane protège les CSI du stress oxydatif, le butyrate a un effet cytostatique sur ces cellules. Une meilleure compréhension des mécanismes moléculaires mis en jeux dans ces régulations devrait permettre de mieux appréhender ces effets apparemment contradictoires. Quels récepteurs spécifiques des CSI, autres que Nod2 ou TLR4, sont impliqués, comment ces signaux sont-ils intégrés au sein des CSI, demeurent des questions ouvertes. De manière intéressante, il semble que la signalisation impliquant les PRR au sein de la cellule souche n'active pas une réponse de type inflammatoire, comme c'est le cas dans les autres cellules de l'intestin. L'influence du microbiote sur d'autres aspects de la biologie des CSI comme leur ontogénèse au cours de la maturation de l'intestin, ou leur potentiel de différenciation, reste également à explorer.

L'identification chez la souris d'une flore spécifique des cryptes ouvre un nouveau champ de recherche sur le rôle particulier que pourraient jouer ces bactéries sur l'homéostasie de la crypte. La caractérisation fonctionnelle de ce microbiote, comme l'identification des métabolites libérés par ces bactéries, devraient apporter d'importantes informations sur leur capacité à moduler les propriétés des cellules souches et à décrypter un éventuel rôle protecteur sur ces cellules.

Un enjeu majeur est à présent de comprendre si ces mécanismes sont conservés chez l'homme. Bien que le séquençage du microbiote humain ait permis d'identifier les principaux phylums bactériens présents dans la lumière intestinale, la présence d'une flore spécifique des cryptes chez l'homme, et sa caractérisation fonctionnelle n'ont pas encore été établies. Combinée à la technique des organoïdes intestinaux, désormais applicable au tissu humain, l'identification de ces bactéries devrait permettre d'étudier, au niveau moléculaire, son rôle sur la biologie des cellules souches intestinales humaines.

Enfin, un effet à distance du microbiote intestinal sur les cellules souches hématopoïétiques a été montré [46]. Cette donnée pose donc des questions passionnantes concernant le rôle de ce microbiote dans la régénération d'autres tissus ayant un rapport privilégié avec l'intestin, comme le pancréas ou le foie, mais également la conservation des relations microbiote-cellule souche au niveau d'autres sites corporels comme la peau qui, comme l'intestin, est caractérisée par la présence d'une flore complexe. $\diamond$

\section{SUMMARY}

Microbiota-intestinal stem cells dialog: a key element for intestinal regeneration

The most abundant and well-studied microbiota on the human body resides in the intestinal tract. Its impact extends the limits of the mucosal interface as it plays an essential role in systemic functions such as development of the immune system. At the level of the intestine, commensal microbes play important metabolic functions and promote the integrity of the mucosal barrier. Moreover, a large number of studies points to a role of the microbiota in intestinal regeneration both under homeostatic conditions and after epithelial damage. As intestinal regeneration is sustained by highly proliferative intestinal stem cells (ISCs), these observations raise the question of a direct impact of commensals on the activity of these cells. Key mediators of the dialog between microbes and the epithelium are the immune cells residing in the gut. Consistently, both innate lymphoid cells and macrophages activated by microbial stimuli have been shown to promote ISCs proliferation by secreting cytokines. More direct routes of communication have been described recently, either through the binding of bacterial ligands to Pattern Recognition Receptors expressed in ISCs, or through the sensing by ISCs of bacterial metabolites. In this review, we explore this stem cell-microbiota dialog and its impact on gut homeostasis. $\diamond$

\section{RÉFÉRENCES}

1. Sansonetti PJ. Host-bacteria homeostasis in the healthy and inflamed gut. Curr Opin Gastroenterol 2008 ; $24: 435-9$.

2. Jakobsson $H E$, Piñeiro AR. The composition of the gut microbiota shapes the colon mucus barrier. EMBO Rep 2015 ; 16 : 164-77.

3. Ulluwishewa D, Anderson RC, McNabb WC, et al. Regulation of tight junction permeability by intestinal bacteria and dietary components. J Nutr 2011 ; $141: 769-76$.

4. Yamanaka T, Helgeland L, Farstad IN, et al. Microbial colonization drives lymphocyte accumulation and differentiation in the follicle-associated epithelium of Peyer's patches. J Immunol 2003 ; 170 : 816-22.

5. Clarke TB, Davis KM, Lysenko ES, et al. Recognition of peptidoglycan from the microbiota by Nodl enhances systemic innate immunity. Nat Med 2010 16: 228-31.

6. Khosravi A, Yáñez A, Price JG, et al. Gut microbiota promote hematopoiesis to control bacterial infection. Cell Host Microbe 2014 ; 15:374-81.

7. Shin SC, Kim SH, You H, et al. Drosophila microbiome modulates host developmental and metabolic homeostasis via insulin signaling. Science 2011 ; 334 : 670-4.

8. Schwarzer M, Makki K, Storelli G, et al. Lactobacillus plantarum strain maintains growth of infant mice during chronic undernutrition. Science $2016 ; 351: 854-7$

9. Diaz Heijtz R, Wang S, Anuar F, et al. Normal gut microbiota modulates brain development and behavior. Proc Natl Acad Sci USA 2011 ; 108 : 3047-52.

10. Bauer KC, Huus KE, Finlay BB. Microbes and the mind: emerging hallmarks of the gut microbiota-brain axis. Cell Microbiol 2016; 18 : 632-44.

11. Sekirov I, Russell SL, Antunes LCM, et al. Gut microbiota in health and disease. Physiol Rev $2010 ; 90: 859-904$.

12. Arora T, Bäckhed F. The gut microbiota and metabolic disease: current understanding and future perspectives. J Intern Med $2016 ; 280: 339-49$.

13. Clevers $H$. The intestinal crypt, a prototype stem cell compartment. Cell $2013 ; 154: 274-84$.

14. Tan DWM, Barker N. Intestinal stem cells and their defining niche. Curr Top Dev Biol 2014 ; 107 : 77-107.

15. Sato T, Vries RG, Snippert HJ, et al. Single Lgr5 stem cells build crypt-villus structures in vitro without a mesenchymal niche. Nature $2009 ; 459: 262-5$

16. Kawai T, Akira S. Toll-like receptors and their crosstalk with other innate receptors in infection and immunity. Immunity 2011 ; $34: 637-50$.

17. Rakoff-Nahoum S, Paglino J, Eslami-Varzaneh F, et al. Recognition of commensal microflora by toll-like receptors is required for intestinal homeostasis. Cell $2004 ; 118: 229-41$. 


\section{RÉFÉRENCES}

18. Pull SL, Doherty JM, Mills JC, et al. Activated macrophages are an adaptive element of the colonic epithelial progenitor niche necessary for regenerative responses to injury. Proc Natl Acad Sci USA $2005 ; 102: 99-104$.

19. Nigro G, Rossi R, Commere PH, et al. The cytosolic bacterial peptidoglycan sensor Nod2 affords stem cell protection and links microbes to gut epithelial regeneration. Cell Host Microbe 2014 ; $15: 792-8$.

20. Zanello G, Goethel A, Rouquier $S$, et al. The cytosolic microbial receptor Nod2 regulates small intestinal crypt damage and epithelial regeneration following T cell-induced enteropathy. J Immunol $2016 ; 197: 345-55$.

21. Swanson PA, Kumar A, Samarin S, et al. Enteric commensal bacteria potentiate epithelial restitution via reactive oxygen species-mediated inactivation of focal adhesion kinase phosphatases. Proc Natl Acad Sci USA $2011 ; 108: 8803-8$.

22. Alam M, Midtvedt T, Uribe A. Differential cell kinetics in the ileum and colon of germfree rats. Scand J Gastroenterol 1994 ; 29 : 445-51.

23. Reikvam DH, Erofeev A, Sandvik A, et al. Depletion of murine intestinal microbiota: effects on gut mucosa and epithelial gene expression. PLoS One $2011 ; 6$ : el7996.

24. Cheesman SE, Neal JT, Mittge $\varepsilon$. Epithelial cell proliferation in the developing zebrafish intestine is regulated by the Wnt pathway and microbial signaling via Myd88. Proc Natl Acad Sci USA 2011; 108 (suppl 1): 4570-7.

25. Bonfini A, Liu X, Buchon N. From pathogens to microbiota: how drosophila intestinal stem cells react to gut microbes. Dev Comp Immunol $2016 ; 64: 22-38$.

26. Wong CNA, Ng P, Douglas AE. Low-diversity bacterial community in the gut of the fruitfly Drosophila melanogaster. Environ Microbiol $2011 ; 13$ : 1889-900.

27. Micchelli CA, Perrimon N. Evidence that stem cells reside in the adult drosophila midgut epithelium. Nature $2006 ; 439: 475-9$

28. Jiang $\mathrm{H}$, Patel $\mathrm{PH}$, Kohlmaier $\mathrm{A}$, et al. Cytokine/Jak/Stat signaling mediates regeneration and homeostasisin the drosophila midgut. Cell $2009 ; 137: 1343-55$.

29. Buchon N, Broderick NA, Chakrabarti S, et al. Invasive and indigenous microbiota impact intestinal stem cell activity through multiple pathways in drosophila. Genes Dev $2009 ; 23$ : 2333-44.

30. Alam A, Leoni $G$, Wentworth CC, et al. Redox signaling regulates commensal-mediated mucosal homeostasis and restitution and requires formyl peptide receptor 1. Mucosal Immunol $2013 ; 7$ : 645-55.

31. Jones RM, Desai C, Darby TM, et al. Lactobacilli modulate epithelial cytoprotection through the Nrf2 pathway. Cell Reports $2015 ; 12: 1217-25$.

32. Lindemans CA, Calafiore M, Mertelsmann AM, et al. Interleukin-22 promotes intestinal-stem-cellmediated epithelial regeneration. Nature $2015 ; 528: 560-4$.

33. Sokol H, Seksik P. The intestinal microbiota in inflammatory bowel diseases: time to connect with the host. Curr Opin Gastroenterol $2010 ; 26: 327-31$

34. Sánchez de Medina F, Romero-Calvo I, Mascaraque C, et al. Intestinal inflammation and mucosal barrier function. Inflamm Bowel Dis $2014 ; 20$ : 2394-404

35. Earle KA, Billings $\mathrm{G}$, Sigal M, et al. Quantitative imaging of gut microbiota spatial organization. Cell Host Microbe $2015 ; 18: 478-88$.

36. Pédron T, Sansonetti P. Commensals, bacterial pathogens and intestinal inflammation: an intriguing ménage à trois. Cell Host Microbe $2008 ; 3: 344-7$.

37. Neal MD, Sodhi CP, Jia H, et al. Toll-like receptor 4 is expressed on intestinal stem cells and regulates their proliferation and apoptosis via the $\mathrm{p} 53$ up-regulated modulator of apoptosis. J Biol Chem $2012 ; 287: 37296-308$
38. Ríos-Covián D, Ruas-Madiedo P, Margolles A, et al. Intestinal short chain fatty acids and their link with diet and human health. Front Microbiol 2016; $7: 2030$

39. Besten den G, van Eunen K, Groen AK, et al. The role of short-chain fatty acids in the interplay between diet, gut microbiota, and host energy metabolism. J Lipid Res $2013 ; 54: 2325-40$.

40. Kaiko GE, Ryu SH, Koues OI, et al. The colonic crypt protects stem cells from microbiota-derived metabolites. Cell $2016 ; 165: 1708-20$.

41. Matsuki T, Pédron T, Regnault B, et al. Epithelial cell proliferation arrest induced by lactate and acetate from Lactobacillus casei and Bifidobacterium breve. PLoS One 2013; 8 : e63053.

42. Dutta D, Dobson AJ, Houtz PL, et al. Regional cell-specific transcriptome mapping reveals regulatory complexity in the adult drosophila midgut. Cell Rep 2015 ; 12 : 346-58

43. Larsson $\varepsilon$, Tremaroli V, Lee $Y S$, et al. Analysis of gut microbial regulation of host gene expression along the length of the gut and regulation of gut microbial ecology through MyD88. Gut 2012 ; 61 : 1124-31.

44. Alenghat T, Osborne LC, Saenz SA, et al. Histone deacetylase 3 coordinates commensal- bacteria-dependent intestinal homeostasis. Nature 2014 ; 504: 153-7.

45. Yu DH, Gadkari M, Zhou $Q$, et al. Postnatal epigenetic regulation of intestinal stem cells requires DNA methylation and is guided by the microbiome. Genome Biol $2015 ; 16: 211$.

46. Burberry A, Zeng MY, Ding L, et al. Infection mobilizes hematopoietic stem cells through cooperative NOD-like receptor and Toll-like receptor signaling. Cell Host Microbe 2014 ; 15 : 779-91.

47. Gaboriau-Routhiau V, Cerf-Bensussan N. Microbiote intestinal et développement du système immunitaire. Med Sci (Paris) 2016 ; 32 : 961-7.

48. Barbotin AL, Giacobini P, Prévot V. Le microbiote intestinal : clé de voûte entre l'obésité maternelle et les troubles de la socialisation chez la descendance. Med Sci (Paris) 2016; $32: 930-2$.

49. Romagnolo B. Une relation Paneth entre cellules souches et niche intestinale. Med Sci (Paris) 2012 ; 28 : 1058-60.

50. Storelli G, Leulier F. Croissance, sous-nutrition et microbiote : l'effet bénéfique de souches de Lactobacilles est conservé de la drosophile à la souris. Med Sci (Paris) 2016; 32 : 925-9.

51. Lamas B, Richard ML, Sokol H. CARD9 et colite : un pont entre dysbiose et immunité. Med Sci (Paris) 2016 ; 32 : 933-6.

52. Rahmouni 0 , Dubuquoy L, Desreumaux P, Neut C. Microbiote intestinal et développement des maladies inflammatoires chroniques de l'intestin. Med Sci (Paris) 2016; $32: 968-73$.

53. Burcelin R, Nicolas S, Blasco-Baque V. Microbiotes et maladies métaboliques: de nouveaux concepts pour de nouvelles stratégies thérapeutiques. Med Sci (Paris) 2016 ; 32 : 952-60.

\section{TIRÉS À PART}

P.J. Sansonetti

\begin{tabular}{|c|c|}
\hline $\begin{array}{l}\text { SOCIÉTÉ FRANÇAISE DE CARCINOLOGIE CERVICO.FACIALE } \\
\text { XIV CONGRES }\end{array}$ & $\begin{array}{l}\text { Bon de commande } \\
\text { À retourner à EDK, 109, avenue Aristide Briand - } 92541 \text { Montrouge Cedex } \\
\text { Tél. : } 0141177405 \text { - Fax : } 0149850345 \text { - E-mail : francois.flori@edpsciences.org }\end{array}$ \\
\hline Cancers de l'hypopharynx & $\begin{array}{l}\text { NOM : } \\
\text { Adresse : }\end{array}$ \\
\hline $\begin{array}{l}\text { Carcinomes épidermoìdes de } \\
\text { la pyramide nasale }\end{array}$ & Code postal : \\
\hline & 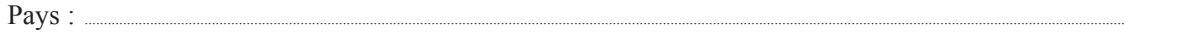 \\
\hline & 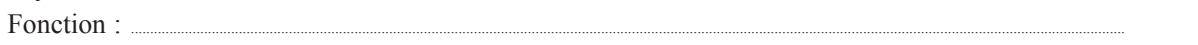 \\
\hline e & $\begin{array}{l}\text { Je souhaite recevoir l'ouvrage Cancers de l'hypopharynx - Carcinomes épidermoïdes de la pyramide nasale : } \\
35 €+3 € \text { de port }=\mathbf{3 8} € \text { TTC }\end{array}$ \\
\hline & 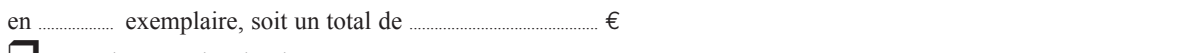 \\
\hline 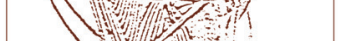 & $\square$ Par chèque, à l'ordre de EDP Sciences \\
\hline \multirow{3}{*}{$\begin{array}{l}\text { Syliatin Moriniere } \\
\text { Patrice Beutter } \\
\text { EDK. }\end{array}$} & $\square$ Par carte bancaire : $\quad \square$ Visa $\square$ Eurocard/Mastercard \\
\hline & 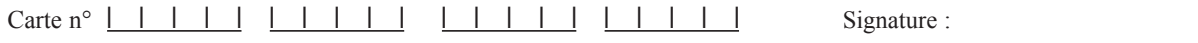 \\
\hline & $N^{\circ}$ de contrôle au dos de la carte : $\quad \begin{array}{lll}\mid & \mid & \mid\end{array}$ \\
\hline
\end{tabular}

